\title{
Quantification of 3D microstructural parameters of trabecular bone is affected by the analysis software
}

Citation for published version (APA):

Mys, K., Varga, P., Stockmans, F., Gueorguiev, B., Wyers, C. E., van den Bergh, J. P. W., \& van Lenthe, G. H. (2021). Quantification of 3D microstructural parameters of trabecular bone is affected by the analysis software. Bone, 142, [115653]. https://doi.org/10.1016/j.bone.2020.115653

Document status and date:

Published: 01/01/2021

DOI:

10.1016/j.bone.2020.115653

Document Version:

Publisher's PDF, also known as Version of record

Document license:

Taverne

Please check the document version of this publication:

- A submitted manuscript is the version of the article upon submission and before peer-review. There can be important differences between the submitted version and the official published version of record.

People interested in the research are advised to contact the author for the final version of the publication, or visit the DOI to the publisher's website.

- The final author version and the galley proof are versions of the publication after peer review.

- The final published version features the final layout of the paper including the volume, issue and page numbers.

Link to publication

\footnotetext{
General rights rights.

- You may freely distribute the URL identifying the publication in the public portal. please follow below link for the End User Agreement:

www.umlib.nl/taverne-license

Take down policy

If you believe that this document breaches copyright please contact us at:

repository@maastrichtuniversity.nl

providing details and we will investigate your claim.
}

Copyright and moral rights for the publications made accessible in the public portal are retained by the authors and/or other copyright owners and it is a condition of accessing publications that users recognise and abide by the legal requirements associated with these

- Users may download and print one copy of any publication from the public portal for the purpose of private study or research.

- You may not further distribute the material or use it for any profit-making activity or commercial gain

If the publication is distributed under the terms of Article $25 \mathrm{fa}$ of the Dutch Copyright Act, indicated by the "Taverne" license above, 
Full Length Article

\title{
Quantification of 3D microstructural parameters of trabecular bone is affected by the analysis software
}

\author{
Karen Mys ${ }^{\mathrm{a}, \mathrm{b}, *}$, Peter Varga ${ }^{\mathrm{b}}$, Filip Stockmans ${ }^{\mathrm{c}}$, Boyko Gueorguiev ${ }^{\mathrm{b}}$, Caroline E. Wyers ${ }^{\mathrm{d}, \mathrm{e}}$, \\ Joop P.W. van den Bergh ${ }^{\mathrm{d}, \mathrm{e}, \mathrm{f}}$, G. Harry van Lenthe ${ }^{\mathrm{a}}$ \\ ${ }^{a}$ Biomechanics Section, Mechanical Engineering, KU Leuven, Leuven, Belgium \\ ${ }^{\mathrm{b}}$ AO Research Institute Davos, Davos, Switzerland \\ ${ }^{\mathrm{c}}$ Muscles \& Movement, Department of Development and Regeneration, KU Leuven Campus Kulak, Kortrijk, Belgium \\ ${ }^{\mathrm{d}}$ Department of Internal Medicine, VieCuri Medical Center, Venlo, the Netherlands \\ ${ }^{\text {e } N U T R I M ~ S c h o o l ~ f o r ~ N u t r i t i o n ~ a n d ~ T r a n s l a t i o n a l ~ R e s e a r c h ~ i n ~ M e t a b o l i s m, ~ M a a s t r i c h t ~ U n i v e r s i t y, ~ M a a s t r i c h t, ~ t h e ~ N e t h e r l a n d s ~}$ \\ ${ }^{\mathrm{f}}$ Department of Internal Medicine, Subdivision of Rheumatology, Maastricht University Medical Centre, Maastricht, the Netherlands
}

\section{A R T I C L E I N F O}

\section{Keywords:}

Quantification

Microstructural parameters

Analysis software

\begin{abstract}
A B S T R A C T
Over the last decades, the use of high-resolution imaging systems to assess bone microstructural parameters has grown immensely. Yet, no standard defining the quantification of these parameters exists. It has been reported that different voxel size and/or segmentation techniques lead to different results. However, the effect of the evaluation software has not been investigated so far. Therefore, the aim of this study was to compare the bone microstructural parameters obtained with two commonly used commercial software packages, namely IPL (Scanco, Switzerland) and CTan (Bruker, Belgium). We hypothesized that even when starting from the same segmented scans, different software packages will report different results.

Nineteen trapezia and nineteen distal radii were scanned at two resolutions $(20 \mu \mathrm{m}$ voxel size with microCT and HR-pQCT $60 \mu \mathrm{m}$ ). The scans were segmented using the scanners' default protocol. The segmented images were analyzed twice, once with IPL and once with CTan, to quantify bone volume fraction (BV/TV), trabecular thickness (Tb.Th), trabecular separation (Tb.Sp), trabecular number (Tb.N) and specific bone surface (BS/BV).

Only small differences between IPL and CTan were found for BV/TV. For Tb.Th, Tb.Sp and BS/BV high correlations $\left(R^{2} \geq 0.99\right)$ were observed between the two software packages, but important relative offsets were observed. For microCT scans, the offsets were relative constant, e.g., around $15 \%$ for Tb.Th. However, for the HR-pQCT scans the mean relative offsets ranged over the different bone samples (e.g., for Tb.Th from $14.5 \%$ to $19.8 \%)$. For Tb.N, poor correlations $\left(0.43 \leq R^{2} \leq 0.81\right)$ for all tested cases were observed.

We conclude that trabecular bone microstructural parameters obtained with IPL and CTan cannot be directly compared except for BV/TV. For Tb.Th, Tb.Sp and BS/BV, correction factors can be determined, but these depend on both the image voxel size and specific anatomic location. The two software packages did not produce consistent data on Tb.N. The development of a universal standard seems desirable.
\end{abstract}

\section{Introduction}

The use of high-resolution imaging systems to assess bone microstructural parameters in animals and humans has grown immensely during the last decades $[1,2]$. Several commercial scanners are on the market, each one with its own analysis software. Yet, no standard exists either for quantification of the morphological measures, or for calibration of the analysis software. It has been reported that different voxel size and/or segmentation techniques lead to different results and hence that it is not possible to compare the absolute values of bone parameters obtained from different voxel size scans and/or segmentation techniques [3-6].

In commercially available scanners, two voxel size ranges are commonly used for imaging and quantification of bone microstructural parameters for human beings. For ex vivo analyses, a voxel size of $10-20 \mu \mathrm{m}$ is commonly accepted [7] as achieved by micro-computed tomography (microCT). State-of-the art human in vivo scanners can reach a somewhat lower resolution. For in vivo investigations, the high resolution peripheral computed tomography (HR-pQCT) technology offers the highest resolution, with the XtremeCT-II scanner (Scanco

\footnotetext{
* Corresponding author at: AO Research Institute Davos, Davos, Switzerland.

E-mail address: karen.mys@aofoundation.org (K. Mys).
} 


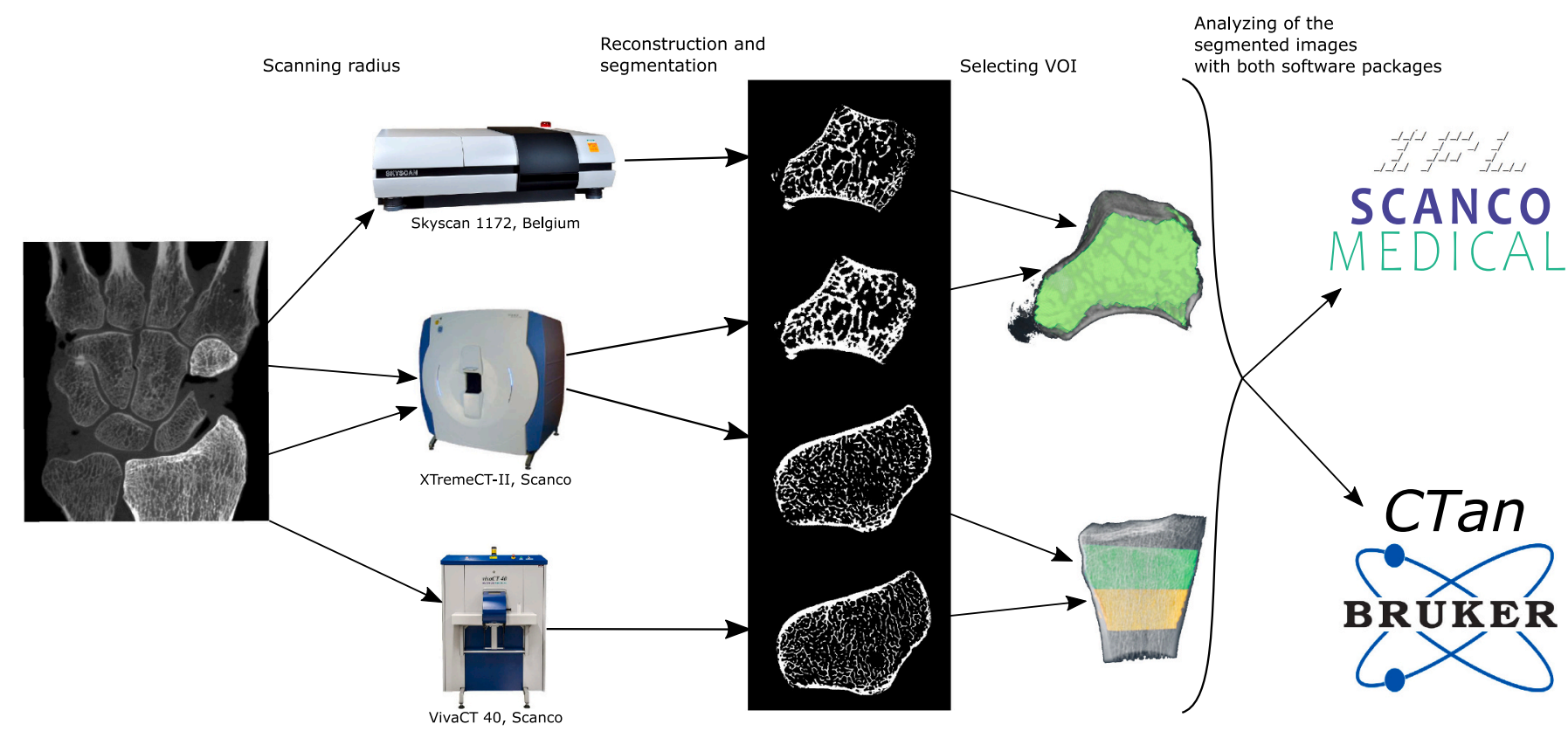

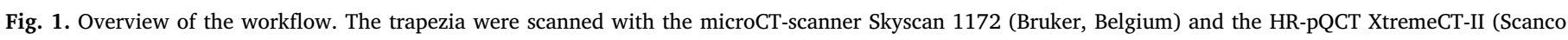

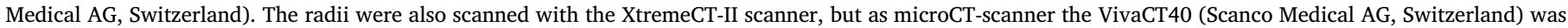

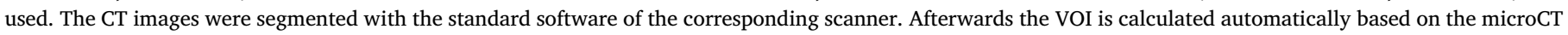

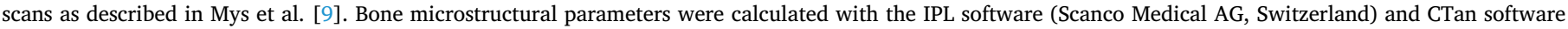
(Bruker, Belgium).

Medical AG, Switzerland) providing a reconstructed isotropic voxel size of $60.7 \mu \mathrm{m}$. Next to HR-pQCT, cone-beam computed tomography (CBCT) can be used for high-resolution in vivo imaging, but at a slightly lower resolution ( $75 \mu \mathrm{m}$ voxel size for the NewTom $5 \mathrm{G}$ scanner (Cefla, Italy)) [8].

Several software packages exist for a quantitative evaluation of the images acquired by the scanners. Thus far, the results delivered by those software packages have not been subjected to direct comparison. Nevertheless, this comparison would be important to correctly interpret and compare the results of various research studies. Hence, the aim of this study was to compare the bone microstructural parameters calculated with different software packages. We opted to compare the two most widely used commercial software packages, more specifically, the Image Processing Language (IPL) software implemented in Scanco systems [9] and the CTan software delivered with Bruker scanners $[10,11]$. The second aim was to evaluate the effect that different voxel size and anatomical locations could have on the comparison. We hypothesized that, even when starting from the same segmented images, the results of the two analysis software packages would show differences in the calculated bone microstructural parameters due to different implementations and quantification methods.

\section{Materials \& methods}

\subsection{Sample collection}

The software comparison was performed on two different bones of the wrist, namely the trapezium, being one of the carpal bones, and the distal radius that is the most commonly investigated peripheral site in osteoporosis research. Two sets of human cadaveric bone samples had been collected in previous studies $[9,10]$. In short, nineteen trapezia (18 female, 1 male; 7 left, 12 right) of severe osteoarthritic patients were harvested via trapeziectomy by a hand surgeon (FS) in the hospital AZ Groeninge (Kortrijk, Belgium). The age of the donors ranged from 53 to 76 years (mean \pm standard deviation (SD): $63.4 \pm 7.0$ years). All donors consented to use their data and their extracted specimen for anonymous research use. No extra medical interventions were done for this study. In addition, nineteen cadaveric radii (14 females, 5 males; 8 left, 11 right) were obtained from Science Care (USA). Donor age ranged from 25 to 93 years (mean \pm SD: $67.9 \pm 16.2$ year). All samples were conserved fresh-frozen at $-20^{\circ}$ Celsius.

\subsection{Image acquisition and processing}

Each specimen was scanned twice: at a high resolution close to $20 \mu \mathrm{m}$ voxel size and at a moderate resolution close to $60 \mu \mathrm{m}$ voxel size (Fig. 1). Specifically, the trapezia were scanned at a voxel size of 19.84 $\mu \mathrm{m}$ using an ex vivo microCT-scanner (Skyscan 1172, Bruker, Belgium), and at a voxel size of $60.7 \mu \mathrm{m}$ using a HR-pQCT device (XtremeCT-II, Scanco Medical AG, Switzerland). The radii were scanned at voxel sizes of $19 \mu \mathrm{m}$ and $60.7 \mu \mathrm{m}$ using a small animal microCT scanner (VivaCT40, Scanco Medical AG, Switzerland) and the XtremeCT-II, respectively. All images were reconstructed and segmented using the standard software and the default settings of the respective scanner [8-10].

For the trapezia, the whole trabecular bone compartment was used for the analyses. For the radii, two $9 \mathrm{~mm}$ sections were defined as volumes of interest. The first section was selected strictly adjacent to the most proximal point of the subchondral endplate and perpendicular to the long bone axis and will be addressed as 'subchondral section' in this paper. The second section was selected directly distal to the first section and corresponded closely to the measurement area recommended for clinical scanning, termed 'standard section' throughout this work. The trabecular volumes of interest (VOI) were selected automatically on the microCT or vivaCT images using an in-house $\mathrm{C}++$ script which was based on a method developed by Buie et al. $[9,11]$. The corresponding VOIs of the HR-pQCT images were identified by image registration and transferring the microCT masks to the HR-pQCT scans. In more detail, the HR-pQCT images were spatially registered to the microCT images by a rigid voxel-based gray value registration in AMIRA software package (v6.2, Thermo Fisher Scientific, USA). The obtained transformation matrix was used to transform the microCT mask to the HR-pQCT 
Table 1

Specific commands, the technique where the implementation is based on as well as the companies explanation about the calculated bone microstructural parameters bone volume fraction (BV/TV and BV/TV*), trabecular thickness (Tb.Th), trabecular separation (Tb.Sp), trabecular number (Tb.N) and specific bone surface (BS/BV) and this for the software packages IPL (Scanco Medical AG, Switzerland) and CTan (Bruker, Belgium). The voxel counting technique was used in IPL to calculate BV/ $\mathrm{TV}$, while marching cube was used to calculate BV/TV*.

\begin{tabular}{|c|c|c|c|}
\hline & & IPL (Scanco Medical AG) & CTan (Bruker) \\
\hline \multirow{3}{*}{$\begin{array}{l}\mathrm{BV} / \mathrm{TV} \\
\text { Bone volume } \\
\text { fraction }\end{array}$} & Function: & $\begin{array}{l}\text { vox_scanco_param and tri_da_metric for MicroCT or vox_scanco_param } \\
\text { for XtremeCT-II }\end{array}$ & 3D analysis \\
\hline & Technique: & $\begin{array}{l}\text { Voxel counting (vox_scanco_param) and marching cube } \\
\text { (tri_da_metric_db) [14]. We named the latter BV/TV* }\end{array}$ & Marching cube [14] \\
\hline & Manual: & No explanation & $\begin{array}{l}\text { Total volume of each binarized 3D object within the VOI divided } \\
\text { by the total volume of the VOI. The 3D volume measurement is } \\
\text { based on the marching cube volume model. }\end{array}$ \\
\hline \multirow{3}{*}{$\begin{array}{l}\text { Tb.Th } \\
\text { Trabecular } \\
\text { thickness }\end{array}$} & Function: & dt_object_param for MicroCT or dt_thickness for XtremeCT-II & $3 \mathrm{D}$ analysis \\
\hline & Technique: & Distance transform [15] & Distance transform [15] \\
\hline & Manual: & $\begin{array}{l}\text { The mean thickness of the structure is calculated with the distance } \\
\text { transformation (DT) method by filling largest spheres into the object } \\
\text { and calculating their mean diameter (volume weighted mean). }\end{array}$ & $\begin{array}{l}\text { Local thickness definition is based on the definition of } \\
\text { Hildebrand and Ruegsegger [15]. They defined local thickness } \\
\text { for a point in solid as the diameter of the largest sphere which } \\
\text { fulfills two conditions: } \\
\text { - The sphere encloses the point (but the point is not necessarily } \\
\text { the center of the sphere) } \\
\text { - The sphere is entirely bounded within the solid surfaces. } \\
\text { The base of the implementation is the distance transform method } \\
\text { described by Remy and Thiel [20]. }\end{array}$ \\
\hline \multirow{3}{*}{$\begin{array}{l}\text { Tb.Sp } \\
\text { Trabecular } \\
\text { separation }\end{array}$} & Function: & dt_background_param for MicroCT or dt_spacing for XtremeCT-II & 3D analysis \\
\hline & Technique: & Distance transform [15] & Distance transform [15] \\
\hline & Manual: & $\begin{array}{l}\text { The mean separation of the structure is calculated with the distance } \\
\text { transformation (DT) method by filling largest spheres into the } \\
\text { background of the object and calculating their mean diameter (volume } \\
\text { weighted mean). }\end{array}$ & $\begin{array}{l}\text { Local separation is based on the definition of Hildebrand and } \\
\text { Ruegsegger [15] as for Tb.Th, but this time the technique is } \\
\text { applied on the background of the object. The base of the } \\
\text { implementation is the distance transform method described by } \\
\text { Remy and Thiel [20]. }\end{array}$ \\
\hline \multirow{4}{*}{$\begin{array}{l}\text { Tb.N } \\
\text { Trabecular } \\
\text { number }\end{array}$} & Function: & dt_mat_param & 3D analysis \\
\hline & & $\begin{array}{l}\text { Scanco named this parameter Tb.N*. This is the standard used } \\
\text { definition for calculating Tb.N. }\end{array}$ & \\
\hline & Technique: & Distance transform [15] & Parallel plate mode \\
\hline & Manual: & $\begin{array}{l}\text { The mean trabecular number of the structure is calculated with the } \\
\text { distance transformation (DT) method by filling largest spheres into the } \\
\text { background of the mid axis transformed object and calculating their } \\
\text { mean diameter (volume weighted mean). }\end{array}$ & $\begin{array}{l}\text { The mean trabecular number is calculated by application of the } \\
\text { equation of the parallel plate model (fractional volume/ } \\
\text { thickness), but using a direct 3D measurement of thickness. } \\
\text { Tb.N = (BV/TV)/Tb.Th (plate model) }\end{array}$ \\
\hline \multirow{3}{*}{$\begin{array}{l}\text { BS/BV } \\
\text { Specific bone } \\
\text { surface }\end{array}$} & Function: & tri_da_metric_db & 3D analysis \\
\hline & Technique: & Marching cube $[14]$ & Marching cube [14] \\
\hline & Manual: & $\begin{array}{l}\text { The 3D surface measurement is based on triangulation (marching cubes } \\
\text { model) of the segmented object. }\end{array}$ & $\begin{array}{l}\text { The 3D surface measurement is based on the marching cubes } \\
\text { model of the segmented object. }\end{array}$ \\
\hline
\end{tabular}

Table 2

Specific values of the arguments used in the IPL-commands (Scanco Medical AG, Switzerland). For all commands, the values recommended by Scanco for analyzing the distal radius were used. The explanation of all commands can be found in the Scanco Manual [19].

\begin{tabular}{|c|c|c|c|c|c|c|c|c|c|}
\hline & & vox_scanco_param & dt_object_param & dt_thickness & dt_background_param & dt_spacing & dt_mat_param & dt_number & tri_da_metric_db \\
\hline \multirow{10}{*}{ Parameters } & roi_radius_factor & / & 10,000 & / & 10,000 & / & 10,000 & / & / \\
\hline & ridge_epsilon & I & 0.9 & 0.9 & 0.9 & 0.9 & 0.9 & 0.9 & I \\
\hline & ip_sigma & / & / & / & / & / & / & / & 2 \\
\hline & ip_support & / & I & / & / & / & / & I & 1 \\
\hline & ip_threshold & I & I & / & I & I & / & / & 64 \\
\hline & interpolate & / & / & / & / & / & / & / & TRUE \\
\hline & epsilon & / & / & / & I & / & / & I & 1.2 \\
\hline & edges & / & / & / & / & / & / & / & FALSE \\
\hline & suppress_boundary & / & / & 2 & / & 2 & / & 2 & / \\
\hline & version & / & I & 3 & / & 3 & / & 3 & / \\
\hline \multirow[t]{3}{*}{ Used for } & MicroCT & $\mathrm{x}$ & $\mathrm{x}$ & & $\mathrm{x}$ & & $\mathrm{x}$ & & $\mathrm{x}$ \\
\hline & XtremeCT-II & $\mathrm{x}$ & & $\mathrm{x}$ & & $\mathrm{x}$ & & $\mathrm{x}$ & \\
\hline & Parameter & $\mathrm{BV} / \mathrm{TV}$ & Tb.Th & Tb.Th & Tb.Sp & Tb.Sp & Tb.N & Tb.N & $\begin{array}{l}\mathrm{BS} / \mathrm{TV} \\
\mathrm{BV} / \mathrm{TV}^{*}\end{array}$ \\
\hline
\end{tabular}

images in Matlab R2016a (The Mathworks, United States).

\subsection{Calculation of trabecular bone microstructural parameters}

Starting from the segmented images, bone microstructural parameters were calculated with the IPL software (Scanco Medical AG) as well as with the software CTan (Bruker). It is important to note that, for a given specimen and scan resolution/voxel size, both software packages were provided with the same segmented images. For both software packages, the standard 3D direct analysis method was used. 
Table 3

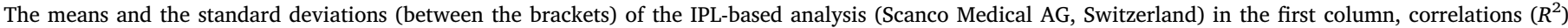

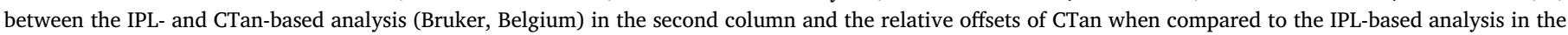

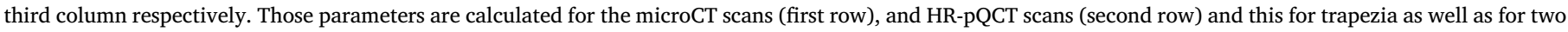

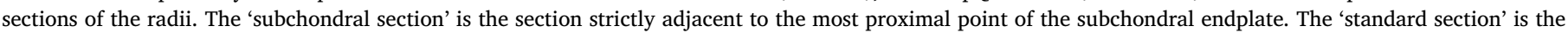
section proximal from 'subchondral section' and corresponds to the normal patient measurement area.

\begin{tabular}{|c|c|c|c|c|c|c|c|c|c|c|}
\hline & & \multicolumn{3}{|l|}{ Trapezium } & \multicolumn{3}{|c|}{ Radius subchondral section } & \multicolumn{3}{|c|}{ Radius standard section } \\
\hline & & Mean IPL & $\mathrm{R}^{2}$ & Relative offset & Mean IPL & $\mathrm{R}^{2}$ & Relative offset & Mean IPL & $\mathrm{R}^{2}$ & Relative offset \\
\hline \multirow{6}{*}{ MicroCT scans } & BV/TV (\%) & $23.41(2.75)$ & 1.00 & $-0.18 \%$ & $16.03(3.36)$ & 1.00 & $-0.28 \%$ & $12.45(3.89)$ & 1.00 & $-0.37 \%$ \\
\hline & $\mathrm{BV} / \mathrm{TV}^{*}(\%)$ & $23.75(2.84)$ & 1.00 & $-1.61 \%$ & $16.23(3.44)$ & 1.00 & $-1.51 \%$ & $12.58(3.97)$ & 1.00 & $-1.37 \%$ \\
\hline & Tb.Th (mm) & $0.16(0.02)$ & 1.00 & $14.76 \%$ & $0.15(0.01)$ & 1.00 & $15.04 \%{ }^{*}$ & $0.15(0.01)$ & 1.00 & $14.89 \%{ }^{*}$ \\
\hline & Tb.Sp (mm) & $0.58(0.07)$ & 0.99 & $4.99 \%$ & $0.70(0.11)$ & 1.00 & $6.30 \%$ & $0.82(0.18)$ & 1.00 & $4.64 \%$ \\
\hline & Tb.N (1/mm) & $1.78(0.18)$ & 0.66 & $-26.70 \%$ & $1.45(0.22)$ & 0.79 & $-35.00 \%$ & $1.26(0.26)$ & 0.81 & $-42.06 \%$ \\
\hline & $\mathrm{BS} / \mathrm{BV}(1 / \mathrm{mm})$ & $16.67(1.97)$ & 0.99 & $24.30 \% *$ & $17.76(1.71)$ & 0.99 & $18.49 \% *$ & $18.29(1.85)$ & 1.00 & $19.08 \% *$ \\
\hline \multirow[t]{4}{*}{ XtremeCT scans } & BV/TV (\%) & $35.67(4.67)$ & 1.00 & $-0.75 \%$ & $17.53(4.99)$ & 1.00 & $-2.00 \%$ & $13.72(0.05)$ & 1.00 & $-2.46 \%$ \\
\hline & Tb.Th (mm) & $0.28(0.03)$ & 1.00 & $19.79 \%$ * & $0.21(0.01)$ & 1.00 & $15.07 \%{ }^{*}$ & $0.21(0.02)$ & 1.00 & $14.53 \%$ \\
\hline & Tb.Sp (mm) & $0.60(0.07)$ & 0.98 & $13.18 \%$ & $0.81(0.15)$ & 1.00 & $11.79 \%$ & $0.98(0.30)$ & 1.00 & $8.80 \% *$ \\
\hline & Tb.N $(1 / \mathrm{mm})$ & $1.60(0.15)$ & 0.43 & $-32.46 \%$ & $1.24(0.20)$ & 0.80 & $-44.09 \% *$ & $1.07(0.25)$ & 0.81 & $-48.19 \% *$ \\
\hline
\end{tabular}

* IPL-based parameter and CTan-based parameter are significant different (5\% significance level) following the Kolmogorov-Smirnov test.

The evaluated trabecular bone parameters included bone volume fraction (BV/TV), trabecular thickness (Tb.Th), trabecular separation (Tb.Sp), trabecular number (Tb.N - named Tb.N* in Scanco's user manual) and specific bone surface (BS/BV) [12]. The calculation techniques following the software's manual are summarized in Table 1. For the IPL algorithms, the standard settings as recommended by Scanco were used (Table 2). Remark that Scanco is using different settings for microCT as for XtremeCT-II. Hence, different results will be obtained when evaluating the same data with the microCT or with the XtremeCT-II script. For CTan, there were no adjustable parameters of the evaluation procedure.

\subsection{Statistics}

Statistical analyses were performed in Matlab. To compare trabecular microstructural parameters obtained with both software packages, linear regression analysis was performed. The coefficient of determination $\left.\left(R^{2}=\text { (Pearson correlation coefficient }\right)^{2}\right)$ which describes the goodness of the fit on a line (linear regression) and relative offset (mean $\left.\left(\frac{C T a n-I P L}{I P L}\right)\right)$ which describes the distance from the ideal line through 0 and with a slope of 1 , were evaluated.

Furthermore, trendlines and Bland-Altman plots were generated to assess how well the software packages correspond as well as to assess the influence of the anatomical regions on this correspondence. The two-sample Kolmogorov-Smirnov test was used to evaluate for each bone parameter and for each anatomical region whether the values of IPL and CTan were significantly different at 5\% significance level [13].

\section{Results}

\subsection{MicroCT scans}

Excellent correlation $\left(R^{2}=1\right)$ and only small non-significant differences were observed for the parameter BV/TV between the two software packages, with relative offset ranging from $-0.4 \%$ to $-0.2 \%$ (Table 3, Fig. 2). The offset became slightly larger when also the marching cube technique was used in IPL. For Tb.Th, Tb.Sp and BS/BV, the obtained results correlated well $\left(R^{2} \geq 0.99\right)$ between IPL and CTan, but relative offsets were observed. The results were significantly different for Tb.Th and BS/BV, but not for Tb.Sp. For Tb.Th and Tb.Sp, the relative offsets varied only slightly between the two anatomical locations (from $14.8 \%$ to $15.0 \%$ for $\mathrm{Tb}$.Th and $4.6 \%$ to $6.3 \%$ for $\mathrm{Tb} . \mathrm{Sp}$ ). This was not the case for BS/BV, as the relative offset varied from $18.5 \%$ to $24.3 \%$. For Tb.N, moderate to poor correlations were obtained $\left(0.66 \leq R^{2} \leq 0.81\right)$ and the results were significantly different between the software packages of both anatomical locations.

\subsection{HR-pQCT scans}

Similarly to the microCT images, BV/TV, Tb.Th and Tb.Sp exhibited strong correlations $\left(R^{2} \geq 0.98\right)$, while Tb.N showed poor correlations $\left(0.43 \leq R^{2} \leq 0.81\right)$ (Table 3, Fig. 3). However, the magnitude and range of the relative offsets were in general larger compared to microCT. The relative offsets ranged from $14.5 \%$ to $19.8 \%$ for $\mathrm{Tb}$.Th and from $8.8 \%$ to $13.2 \%$ for Tb.Sp. BV/TV was the only parameter for which the HR-pQCT-based results were not significantly different between the two software packages.

\section{Discussion}

The aim of this study was to compare bone microstructural parameters as calculated by the software packages IPL and CTan, and to assess their agreement at ex vivo microCT resolution $(\sim 20 \mu \mathrm{m}$ voxel size) as well as at in vivo HR-pQCT resolution $(\sim 60 \mu \mathrm{m}$ voxel size). A strength of our approach was that identical segmented images were used when comparing IPL to CTan. This prevented that differences in image segmentation could have affected the outcome of our study as a confound factor; hence, the identified differences in the quantified bone parameters were due only to differences in the software implementations for calculation of those parameters.

The results obtained for BV/TV were highly similar for both software packages $\left(R^{2}=1\right.$ and relative offsets $\leq 2.46 \%$ ), despite the differences in the software implementations. The implementation used in IPL is mainly based on voxel counting, whereas the implementation in CTan is based on the surface mesh model triangulated with the marching cubes algorithm [14]. For microCT, Scanco also implemented a marching cube based algorithm to calculate BV/TV (indicated as BV/ $\mathrm{TV}^{*}$ in this manuscript), however, this did not improve the relative offset between the software packages. High correlations $\left(R^{2} \geq 0.98\right)$ were found for Tb.Th, Tb.Sp and BS/BV, but the obtained absolute values were higher when CTan was used and these differences reached statistical significance for Tb.Th and BS/BV. As both software packages are based on similar theoretical backgrounds (distance transformation for Tb.Th and Tb.Sp, marching cube for BS/BV) for those parameters, we hypothesize that the respective implementations are slightly different. For Tb.N, only poor correlations were obtained; this can be explained by the different methodologies used to calculate this parameter. The IPL implementation utilizes the distance transform [15], whereas CTan's calculation uses the parallel plate model $[16,17]$. It has 

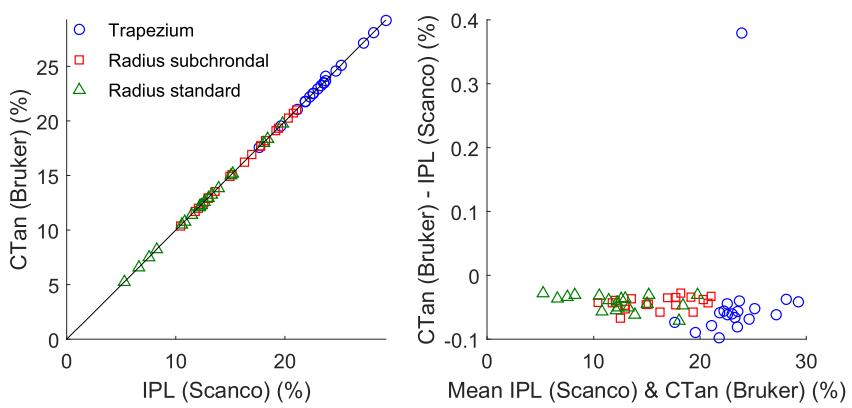

(a) Bone volume fraction $(B V / T V)$
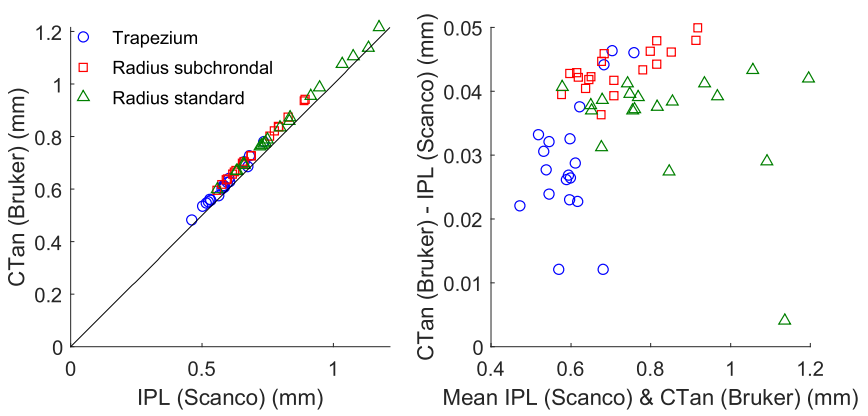

(c) Trabecular separation (Tb.Sp)
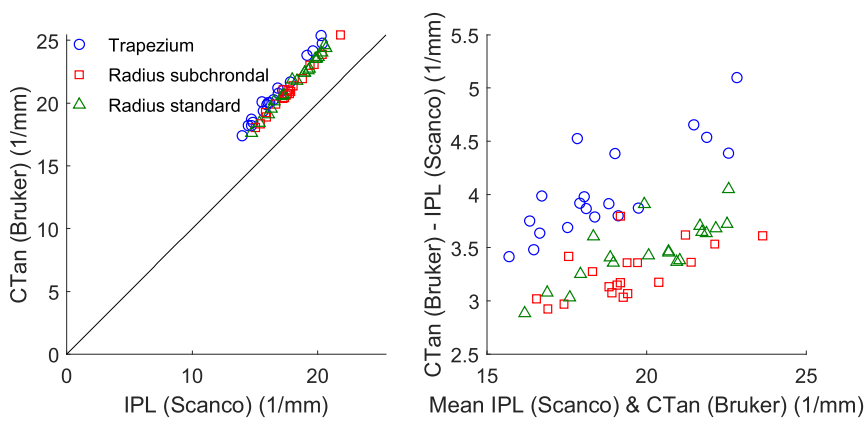

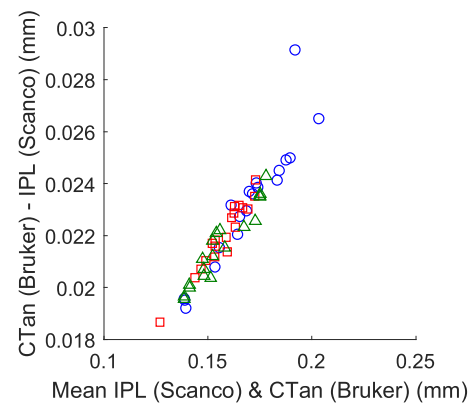

(b) Trabecular thickness (Tb.Th)
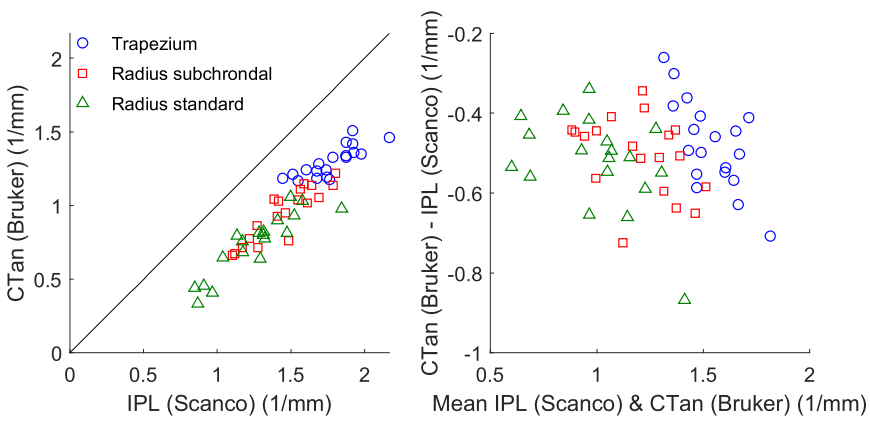

(d) Trabecular number (Tb.N)

\section{(e) $(B S / B V)$}

Fig. 2. Regression plots and Bland Altman plots for the microCT scans analyzed once with IPL (Scanco Medical AG, Switzerland) and CTan (Bruker, Belgium) for bone volume fraction (BV/TV), trabecular thickness (Tb.Th), trabecular separation (Tb.Sp), trabecular number (Tb.N) and specific bone surface (BS/BV). The 'subchondral section' is the section strictly adjacent to the most proximal point of the subchondral endplate. The 'standard section' is the section proximal to the 'subchondral section' and corresponds to the standard patient measurement area. The solid line on the regression plot indicates the line $\mathrm{y}=\mathrm{x}$.

been demonstrated that the parallel plate model introduces a volumedependent offset for Tb.Th and Tb.Sp [18]. We suggest to follow the reporting recommendations made by Bouxsein et al. in 2010 (Guidelines for assessment of bone microstructure in rodents using microcomputed tomography) [12] which are being adopted by many in the research community. They proposed to calculate Tb.N with the distance transform method as the inverse of the mean distance between the midaxis of the structure. This corresponds to the IPL implementation.

For the microCT-based analyses, the relative offsets for Tb.Th and Tb.Sp were fairly constant, ranging from $14.8 \%$ to $15.0 \%$, and from $4.6 \%$ to $6.3 \%$, respectively. This suggests that correction factors could be used to compensate for the different implementations of the analyzing software for those parameters. Remark that those correction factors only compensate for the difference in the analyzing software and do not include other aspects which can cause discrepancies in the results, such as segmentation and voxel size which are explained later in this manuscript. We suggest to use a correction factor of 1.15
$(1+$ relative offset) for Tb.Th and 1.05 for Tb.Sp to convert data obtained with IPL to CTan. For the HR-pQCT-based analyses, relative offsets were higher (ranging from $14.5 \%$ to $19.8 \%$ for Tb.Th and from $8.8 \%$ to $13.2 \%$ for Tb.Sp) and calculated correction factors differed per anatomical location; hence, these need to be determined for each specific anatomical site. These findings for HR-pQCT can be explained by the lower resolution of the scanner, where even small differences in both the implementation procedure and the imaged bone microstructure will have a larger effect on the quantified microstructural parameters.

In this study, we demonstrated that even when starting from the same segmented images, different software packages using default settings calculate significantly different results for bone microstructural parameters. This problem could be solved by standardizing the analysis.

The lack of standardization of the analysis software is not the only reason limiting the direct comparison between absolute values of bone microstructural parameters in various studies. First et al., also the used 


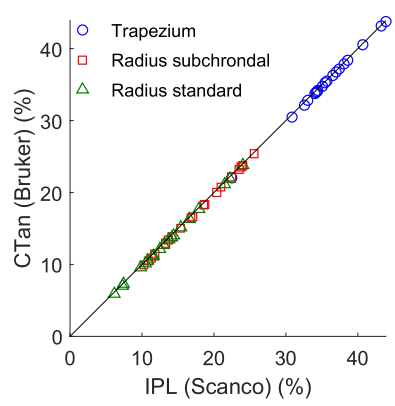

(a) Bone volume fraction $(B V / T V)$
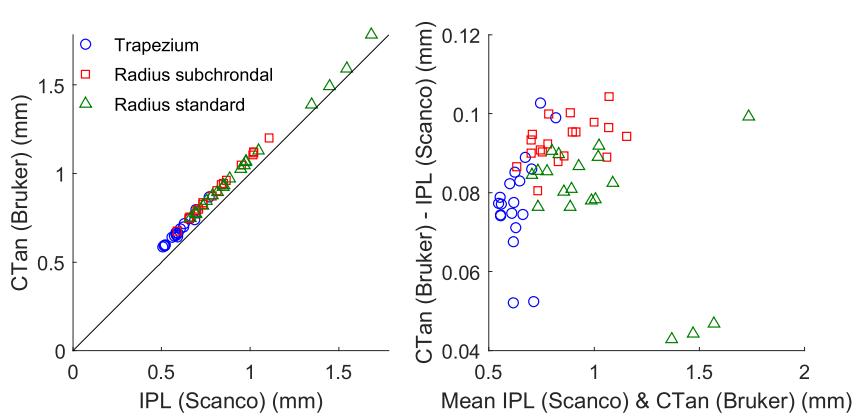

(c) Trabecular separation (Tb.Sp)
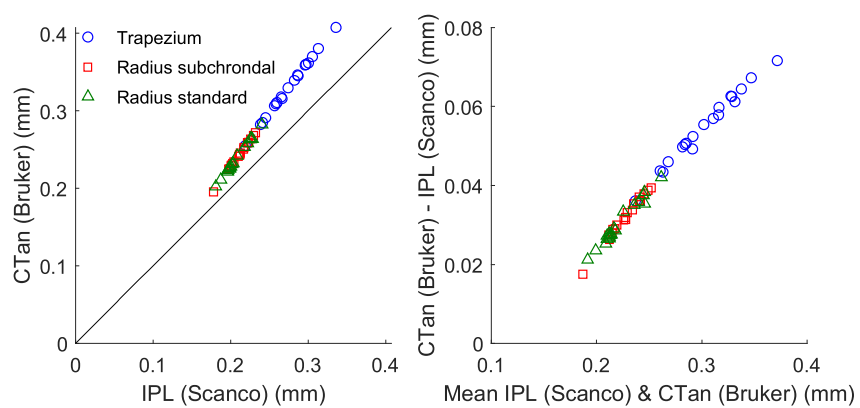

(b) Trabecular thickness (Tb.Th)
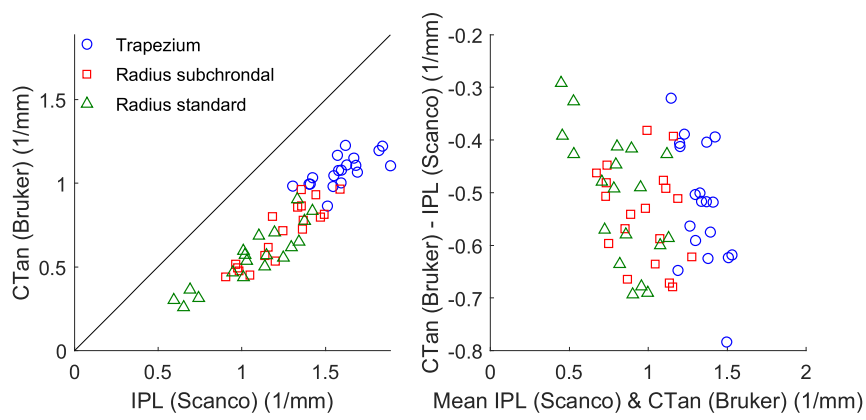

(d) Trabecular number (Tb.N)

Fig. 3. Regression plots and Bland Altman plots for the HR-pQCT scans analyzed once with IPL (Scanco Medical AG, Switzerland) and CTan (Bruker, Belgium) for bone volume fraction (BV/TV), trabecular thickness (Tb.Th), trabecular separation (Tb.Sp) and trabecular number (Tb.N). The 'subchondral section' is the section strictly adjacent to the most proximal point of the subchondral endplate. The 'standard section' is the section proximal to the 'subchondral section' and corresponds to the standard patient measurement area. The solid line on the regression plot indicates the line $\mathrm{y}=\mathrm{x}$.

scanner as well as the scanning protocol will have an important influence. It has e.g. already been thoroughly demonstrated that the image voxel size has a significant influence on the calculated absolute values of the bone microstructural parameters $[3,5,6]$. Standardization of the scanner as well as the scanning protocol should not be aimed for, because these are application specific; e.g., in vivo scanning will typically require short scanning times and low radiation dose, whereas achieving a high level of detail may be critical in an ex vivo scan.

Another factor influencing the absolute values of the bone microstructural parameters is the segmentation procedure. Also here, standardization of the segmentation process is not desired as specific filtering operations and adaptive segmentation techniques may be required to reduce the noise and to improve the contrast.

Also the well-accepted gold standard to calculate bone microstructural parameters, namely microCT [7], is not standardized (e.g., voxel size; segmentation protocol) This scanner is used by most research groups to validate other scanners and segmentation techniques. No standard protocol exists for either the scanner device, the used resolution/voxel size, or the segmentation procedure.

The authors believe that there is an important need for the bone research community to define a 'gold standard' to which scanners and their respective analysis software can be calibrated. This can be achieved by developing bone-like phantoms of trabecular and cortical bone and this of human bone samples as well as of animals (rodents, big animal) with well specified properties to be used for calibration. Like this, researchers and clinicians can use the scanner, segmentation and analysis method of their choice, which allow optimal usage and further innovation of all components, but at the same time parameters are quite well standardized. On short term, the authors advise to use, if possible, the standard methods of the scanners, to calibrate all scans to Hounsfield units and to report in detail scanning, segmentation, analysis methods as well as correction factors (if used). This will not make everything standardized, but this makes it at least reproducible. Specifically, if compared to a gold standard microCT-image, we advise to do this against a $20 \mu \mathrm{m}$ microCT scan which is segmented with a global threshold. The correction values calculated in this paper could be used as correction values to convert from Bruker to Scanco and vice versa. We advise to determine own correction values when using other analysis software.

A limitation of the current study is that our findings may not be representative for other sites and image resolutions/voxel sizes. Moreover, only two commercial software packages were compared in this study. However, these two software packages already show large differences, which demonstrates the need for standardization. A third limitation is the limited sample size subjected in our study. The used specimens may not represent a larger population and thus the results cannot be generalized. Fourth, the current investigation was performed in an ex vivo environment and this may have influenced the results. The image quality is expected to be superior compared to what is achievable under in vivo conditions. Another limitation is that only a restricted set of morphological parameters was analyzed. Other parameters such as connectivity, skeletal muscle index (SMI), etc. were not investigated. This study could still be extended by overcoming the above-mentioned limitations; however, this extension would not alter the core message. With this investigation we intend to highlight the need for standardization to enable comparison of bone microstructural parameters between different scanners and studies in the future.

\section{Conclusion}

With this study we demonstrated that trabecular bone microstructural parameters obtained with IPL and CTan cannot be directly compared except for BV/TV. Good correlations were obtained between the two software packages for Tb.Th, Tb.Sp and BS/BV; however, 
correction factors need to be applied to match their absolute values. General correction factors could be determined with regard to Tb.Th and Tb.Sp for the microCT scans, but not for the HR-pQCT scans. Hence, for in vivo HR-pQCT scanning, we advise to calculate different correction factors for different anatomical locations. The outcomes for Tb.N did not correlate well between IPL and CTan for our tested cases. The development of a universal standard for calculation of bone microstructural parameters seems desirable, not only for calibration of different software packages to each other, but also for compensation of other aspects, such as resolution/voxel size, scanner type and segmentation technique.

\section{CRediT authorship contribution statement}

Karen Mys: Conceptualization, Methodology, Software, Validation, Formal analysis, Investigation, Data curation, Writing - original draft, Writing - review \& editing, Visualization, Project administration, Funding acquisition. Peter Varga: Conceptualization, Investigation, Resources, Data curation, Supervision. Filip Stockmans: Conceptualization, Resources, Supervision. Boyko Gueorguiev: Conceptualization, Resources, Supervision. Caroline E. Wyers: Investigation, Resources. Joop P.W. van den Bergh: Investigation, Resources. G. Harry van Lenthe: Conceptualization, Data curation, Supervision, Project administration, Funding acquisition.

\section{Declaration of competing interest}

Karen Mys, Peter Varga, Filip Stockmans, Boyko Gueorguiev, Caroline E. Wyers, Joop P.W. van den Bergh and G. Harry van Lenthe declare that they have no conflict of interest.

\section{Acknowledgments}

The authors are not compensated and there are no other institutional subsidies, corporate affiliations, or funding sources supporting this study unless clearly documented and disclosed. This research was supported by a travel grant for a long stay abroad by The Research Foundation Flanders (FWO), and by KU Leuven Internal Funding (Grant C24/16/027). The authors would like to thank Dr. Haniyeh Hemmatian (KU Leuven, Leuven, Belgium) for assistance with the microCT-scans and Ursula Eberli (AO Research Institute Davos, Davos, Switzerland) for assistance with the IPL software.

\section{References}

[1] P. Geusens, R. Chapurlat, G. Schett, A. Ghasem-Zadeh, E. Seeman, J. de Jong, J. van den Bergh, High-resolution in vivo imaging of bone and joints: a window to microarchitecture, Nat. Rev. Rheumatol. 10 (2014) 304-313, https://doi.org/10. 1038/nrrheum.2014.23.
[2] A. Grüneboom, L. Kling, S. Christiansen, L. Mill, A. Maier, K. Engelke, H.H. Quick, G. Schett, M. Gunzer, Next-generation imaging of the skeletal system and its blood supply, Nat. Rev. Rheumatol. 15 (2019) 533-549, https://doi.org/10.1038/ s41584-019-0274-y.

[3] W. Tjong, G.J. Kazakia, A.J. Burghardt, S. Majumdar, The effect of voxel size on high-resolution peripheral computed tomography measurements of trabecular and cortical bone microstructure, Med. Phys. 39 (2012) 1893-1903, https://doi.org/10 1118/1.3689813.

[4] B.A. Christiansen, Effect of micro-computed tomography voxel size and segmentation method on trabecular bone microstructure measures in mice, Bone Reports. 5 (2016) 136-140, https://doi.org/10.1016/j.bonr.2016.05.006.

[5] D.G. Kim, G.T. Christopherson, X.N. Dong, D.P. Fyhrie, Y.N. Yeni, The effect of microcomputed tomography scanning and reconstruction voxel size on the accuracy of stereological measurements in human cancellous bone, Bone. 35 (2004) 1375-1382, https://doi.org/10.1016/j.bone.2004.09.007.

[6] M. Kothari, T.M. Keaveny, J.C. Lin, D.C. Newitt, H.K. Genant, S. Majumdar, Impact of spatial resolution on the prediction of trabecular architecture parameters, Bone. 22 (1998) 437-443, https://doi.org/10.1016/S8756-3282(98)00031-3.

[7] R. Müller, H. Van Campenhout, B. Van Damme, G. Van Der Perre, J. Dequeker, T. Hildebrand, P. Rüegsegger, Morphometric analysis of human bone biopsies: a quantitative structural comparison of histological sections and micro-computed tomography, Bone. 23 (1998) 59-66, https://doi.org/10.1016/S8756-3282(98) 00068-4.

[8] K. Mys, P. Varga, B. Gueorguiev, H. Hemmatian, F. Stockmans, G.H. van Lenthe, Correlation between cone-beam computed tomography and high-resolution peripheral computed tomography for assessment of wrist bone microstructure, J. Bone Miner. Res. 34 (2019) 867-874, https://doi.org/10.1002/jbmr.3673.

[9] K. Mys, F. Stockmans, E. Vereecke, G.H. van Lenthe, Quantification of bone microstructure in the wrist using cone-beam computed tomography, Bone. 114 (2018) 206-214, https://doi.org/10.1016/j.bone.2018.06.006.

[10] K. Mys, P. Varga, F. Stockmans, B. Gueorguiev, V. Neumann, O. Vanovermeire, C.E. Wyers, J.P.W. van den Bergh, G.H. van Lenthe, Cone-beam CT as a Fast and Promising Technique to Assess the Microstructure of Distal Radii in Clinical Practice, in: Eur. Soc. Biomech. (2019).

[11] H.R. Buie, G.M. Campbell, R.J. Klinck, J.A. MacNeil, S.K. Boyd, Automatic segmentation of cortical and trabecular compartments based on a dual threshold technique for in vivo micro-CT bone analysis, Bone. 41 (2007) 505-515, https:// doi.org/10.1016/j.bone.2007.07.007.

[12] M.L. Bouxsein, S.K. Boyd, B.A. Christiansen, R.E. Guldberg, K.J. Jepsen, R. Müller, Guidelines for assessment of bone microstructure in rodents using micro-computed tomography, J. Bone Miner. Res. 25 (2010) 1468-1486, https://doi.org/10.1002/ jbmr.141.

[13] F.J. Massey, The Kolmogorov-Smirnov test for goodness of fit, J. Am. Stat. Assoc. 46 (1951) 68-78.

[14] W.E. Lorensen, H.E. Cline, Marching cubes: a high resolution 3D surface construction algorithm, Comput. Graph. (ACM). 21 (1987) 163-169.

[15] T. Hildebrand, P. Rüegsegger, A new method for the model-independent assessment of thickness in three-dimensional images, J. Microsc. 185 (1997) 67-75, https:// doi.org/10.1046/j.1365-2818.1997.1340694.x.

[16] A.M. Parfitt, C.H.E. Mathews, A.R. Villanueva, M. Kleerekoper, B. Frame, D.S. Rao, Relationships between surface, volume, and thickness of iliac trabecular bone in aging and in osteoporosis, J. Clin. Invest. 72 (1983) 1396-1409, https://doi.org/10. $1172 / \mathrm{JCI} 111096$.

[17] A.M. Parfitt, M.K. Drezner, F.H. Glorieux, J.A. Kanis, H. Malluche, P.J. Meunier, S.M. Ott, R.R. Recker, Bone Histomorphometry: standardization of nomenclature, symbols, and units, J. Bone Miner. Res. 2 (1987) 595-610.

[18] J.S. Day, M. Ding, A. Odgaard, D.R. Sumner, I. Hvid, H. Weinans, Parallel plate model for trabecular bone exhibits volume fraction-dependent bias, Bone. 27 (2000) 715-720, https://doi.org/10.1016/S8756-3282(00)00371-9.

[19] S.M. AG, XtremeCT II User's Guide, (2018)

[20] E. Remy, E. Thiel, Medial axis for chamfer distances: computing look-up tables and neighbourhoods in 2D or 3D, Pattern Recogn. Lett. 23 (2002) 649-661, https://doi. org/10.1016/S0167-8655(01)00141-6. 